\title{
Article
}

\section{A Multiplicity Theorem for Superlinear Double Phase Problems}

\author{
Beata Derȩgowska ${ }^{1}$, Leszek Gasiński ${ }^{2, *}$ and Nikolaos S. Papageorgiou ${ }^{3}$ \\ 1 Department of Mathematics, Pedagogical University of Cracow, Podchorazych 2, 30-084 Cracow, Poland; \\ beata.deregowska@up.krakow.pl \\ 2 State Higher Vocational School in Tarnow, Institute of Mathematical and Natural Science, Mickiewicza 8, \\ 33-100 Tarnow, Poland \\ 3 Department of Mathematics, National Technical University, Zografou Campus, 15780 Athens, Greece; \\ npapg@math.ntua.gr \\ * Correspondence: leszek.gasinski@up.krakow.pl
}

check for updates

Citation: Derȩgowska, B.; Gasiński, L.; Papageorgiou, N.S. A Multiplicity Theorem for Superlinear Double Phase Problems. Symmetry 2021, 13 , 1556. https://doi.org/10.3390/ sym13091556

Academic Editor: Alexander Zaslavski

Received: 19 July 2021

Accepted: 13 August 2021

Published: 24 August 2021

Publisher's Note: MDPI stays neutral with regard to jurisdictional claims in published maps and institutional affiliations.

Copyright: (c) 2021 by the authors. Licensee MDPI, Basel, Switzerland. This article is an open access article distributed under the terms and conditions of the Creative Commons Attribution (CC BY) license (https:// creativecommons.org/licenses/by/ $4.0 /)$.
Abstract: We consider a nonlinear Dirichlet problem driven by the double phase differential operator and with a superlinear reaction which need not satisfy the Ambrosetti-Rabinowitz condition. Using the Nehari manifold, we show that the problem has at least three nontrivial bounded solutions: nodal, positive and by the symmetry of the behaviour at $+\infty$ and $-\infty$ also negative.

Keywords: double phase operator; Nehari manifold; superlinear reaction; constant sign and nodal solutions; Musielak-Orlicz spaces

MSC: 35J60; 35J92

\section{Introduction}

Let $\Omega \subseteq \mathbb{R}^{N}$ be a bounded domain (that is, a bounded connected set in the $N$ dimensional Euclidean space) with a smooth boundary $\partial \Omega$. In this paper, we study the following double phase problem

$$
\left\{\begin{array}{l}
-\Delta_{p}^{a} u(z)-\Delta_{q} u(z)=f(z, u(z)) \quad \text { in } \Omega, \\
\left.u\right|_{\partial \Omega}=0,1<q<p<+\infty
\end{array}\right.
$$

(see [1]), with the unknown $u: \Omega \longrightarrow \mathbb{R}$. If $a \in L^{\infty}(\Omega) \backslash\{0\}, a(z) \geqslant 0$ for almost all $z \in \Omega$ and $1<r<+\infty$, then by $\Delta_{r}^{a}$ we denote the weighted $r$-Laplacian differential operator defined by

$$
\Delta_{r}^{a} u=\operatorname{div}\left(a(z)|D u|^{r-2} D u\right) .
$$

If $a \equiv 1$, then we have the usual $r$-Laplacian. In problem $(P)$ we have the sum of two such operators. So, the differential operator (left hand side) in $(P)$ is not homogeneous. The differential operator of $(P)$ is related to the so called "double phase" integral functional defined by

$$
J(u)=\int_{\Omega}\left(a(z)|D u|^{p}+|D u|^{q}\right) d z .
$$

The integrand of this functional is the function

$$
\vartheta(z, y)=a(z)|y|^{p}+|y|^{q} \quad \forall(z, y) \in \Omega \times \mathbb{R}^{N} .
$$

We do not assume that the weight $a$ is bounded away from zero (that is, we do not assume that ess inf $a>0$ ). So, the function $\vartheta(z, \cdot)$ exhibits unbalanced growth, namely we have

$$
|y|^{q} \leqslant \vartheta(z, x) \leqslant c_{0}\left(|y|^{p}+|y|^{q}\right) \quad \text { for a.a. } z \in \Omega \text {, all } y \in \mathbb{R}^{N}
$$


for some $c_{0}>0$. Such functionals were first investigated by Marcellini [2,3] and Zhikov [4,5] in the context of problems of the calculus of variations and of nonlinear elasticity theory. The unbalanced growth of $\vartheta(z, \cdot)$ requires the use of Musielak-Orlicz spaces for the treatment of problem $(P)$. The interest for this kind of problems, was revived recently with the work of Mingione and coworkers who produced interesting interior regularity results for local minimizers of such functionals. We refer to the paper of Baroni-Colombo-Mingione [6] and the references therein. We also mention the very recent work of Ragusa-Tachikawa [7] who extended the interior regularity results to anisotropic double phase functionals. However, a global (that is, up to the boundary) regularity theory for these problems, is not yet available and this makes their study difficult.

The reaction $f(z, x)$ in problem $(P)$ is a Carathéodory function, which is $(p-1)$ superlinear at $+\infty$ and symmetrically at $-\infty$. This problem was examined recently by Gasiński-Papageorgiou [8] and Liu-Dai [9], but under stronger conditions on $f(z, \cdot)$. Here using the Nehari method, we show that problem $(P)$ has at least three nontrivial solutions all with sign information (nodal (that is sign-changing) solution, positive solution and by the symmetry of the behaviour of the data also negative solution) and minimum energy (ground state solutions). The use of the Nehari manifold helps us overcome the difficulties that originate from the fact that for double phase problems we have no global regularity theory and so many of the tools and techniques of "balanced" problems cannot be used. Our multiplicity result improves considerably Theorem 4.9 of Gasiński-Papageorgiou [8] and Theorem 1.4 of Liu-Dai [9]. Normally multiplicity results for superlinear problems are obtained by a combination of critical point theory and Morse theory, see the works of Wang [10] (semilinear equations) and Papageorgiou-Rădulescu [11] (nonlinear, nonhomogeneous equations). The lack of global regularity theory for double phase problems, leads to a different approach based on the Nehari manifold, as this was developed by Brown-Wu [12], Szulkin-Weth [13] and Willem [14]. Other existence and multiplicity results for double phase problems can be found in the works of Gasiński-Winkert [15] (coercive equations), Colasuonno-Squassina [16], Ge-Wang-Lu [17] (eigenvalue problems), Gasiński-Winkert [18], Papageorgiou-Vetro-Vetro [19] (Robin problems). For the nonlinear problems related to the nonlinear frequency shift phenomena we refer to Kalyabin et al. [20] and Sadovnikov et al. [21]. Finally we mention the two recent informative survey articles by Mingione-Rădulescu [1] and Rădulescu [22].

\section{Mathematical Background-The Nehari Manifold}

As we already mentioned in the Introduction, the appropriate functional framework for the analysis of double phase problems, is provided by the so called Musielak-Orlicz spaces. For a comprehensive presentation of the theory of these spaces, we refer to the book of Harjulehto-Hästö [23].

We introduce the following hypotheses (denoted by H1) on the weight $a$ and the exponents $q, p$, which will be used in the sequel.

Hypothesis 1. $a \in C^{0,1}(\bar{\Omega}) \backslash\{0\}$ (that is, $a: \bar{\Omega} \longrightarrow \mathbb{R}$ is nonzero and Lipschitz continuous), $a(z) \geqslant 0$ for all $z \in \Omega, 1<q<p<N$ and $\frac{p}{q}<1+\frac{1}{N}$.

Remark 1. The last condition on the exponents $q, p$ is common in Dirichlet double phase problems and guarantees that the Poincare inequality is valid for the corresponding Musielak-Orlicz-Sobolev space (see Harjulehto-Hästö ([23], p. 138)). Note that the inequality $\frac{p}{q}<1+\frac{1}{N}$ implies that $p<q^{*}$ and as we will see later in this section, this guarantees useful compact embeddings among the relevant spaces.

We consider the Carathéodory integrand

$$
\vartheta(z, x)=a(z)|x|^{p}+|x|^{q}
$$


and let

$$
M(\Omega)=\{u: \Omega \longrightarrow \mathbb{R} \text { measurable }\} .
$$

As usual we identify two such functions which differ only on a Lebesgue-null subset of $\Omega$. Then the Musielak-Orlicz space $L^{\vartheta}(\Omega)$ is defined by

$$
L^{\vartheta}(\Omega)=\left\{u \in M(\Omega): \varrho_{\vartheta}(u)<\infty\right\},
$$

with $\varrho_{\vartheta}$ being the modular function defined by

$$
\varrho_{\vartheta}(u)=\int_{\Omega} \vartheta(z, u(z)) d z
$$

where $\vartheta: \Omega \times \mathbb{R} \longrightarrow \mathbb{R}$ is defined by (1). We equip $L^{\vartheta}(\Omega)$ with the so called "Luxembourg norm"

$$
\|u\|_{\vartheta}=\inf \left\{\mu>0: \varrho_{\vartheta}\left(\frac{u}{\mu}\right) \leqslant 1\right\}
$$

(see Adams [24]). Then $L^{\vartheta}(\Omega)$ becomes a Banach space which is separable and reflexive (in fact uniformly convex). Using the space $L^{\vartheta}(\Omega)$, we can define the corresponding Musielak-Orlicz-Sobolev space $W^{1, \vartheta}(\Omega)$ by

$$
W^{1, \vartheta}(\Omega)=\left\{u \in L^{\vartheta}:|D u| \in L^{\vartheta}(\Omega)\right\} .
$$

Here and in the sequel $D u$ denotes the weak gradient of $u \in L^{\vartheta}(\Omega)$. We equip $W^{1, \vartheta}(\Omega)$ with the norm

$$
\|u\|_{1, \vartheta}=\|u\|_{\vartheta}+\|D u\|_{\vartheta} \quad \forall u \in W^{1, \vartheta}(\Omega),
$$

with $\|D u\|_{\vartheta}=\||D u|\|_{\vartheta}$. We set

$$
W_{0}^{1, \vartheta}(\Omega)={\overline{C_{c}^{\infty}(\Omega)}}^{\|\cdot\|_{1, \vartheta}} .
$$

For this space the Poincaré inequality holds (see Harjulehto-Hästö ([23], p. 138)), that is, there exists $\widehat{c}>0$ such that

$$
\|u\|_{\vartheta} \leqslant \widehat{c}\|D u\|_{\vartheta} \quad \forall u \in W_{0}^{1, \vartheta}(\Omega) .
$$

Therefore on $W_{0}^{1, \vartheta}(\Omega)$ we can consider the equivalent norm

$$
\|u\|=\|D u\|_{\vartheta} \quad \forall u \in W_{0}^{1, \vartheta}(\Omega) .
$$

The spaces $W^{1, \vartheta}(\Omega)$ and $W_{0}^{1, \vartheta}(\Omega)$ are Banach spaces which are separable and reflexive (in fact uniformly convex).

There is a close relation between the norm $\|\cdot\|$ and the modular function $\varrho_{\vartheta}$.

Proposition 1. (a) If $u \neq 0$, then $\|u\|=\mu \Longleftrightarrow \varrho_{\vartheta}\left(\frac{u}{\mu}\right)=1$.

(b) $\|u\|<1$ (resp. $=1,>1) \Longleftrightarrow \varrho_{\vartheta}(u)<1$ (resp. $=1,>1$ ).

(c) $\|u\|<1 \Longrightarrow\|u\|^{p} \leqslant \varrho_{\vartheta}(u) \leqslant\|u\|^{q}$.

(d) $\|u\|>1 \Longrightarrow\|u\|^{q} \leqslant \varrho_{\vartheta}(u) \leqslant\|u\|^{p}$.

(e) $\|u\| \rightarrow 0 \Longleftrightarrow \varrho_{\vartheta}(u) \rightarrow 0$ and $\|u\| \rightarrow+\infty \Longleftrightarrow \varrho_{\vartheta}(u) \rightarrow+\infty$.

Proposition 2. (a) $L^{\vartheta}(\Omega) \subseteq L^{r}(\Omega)$ and $W_{0}^{1, \vartheta}(\Omega) \subseteq W_{0}^{1, r}(\Omega)$ continuously for every $1 \leqslant r \leqslant q$. (b) $W_{0}^{1, \vartheta}(\Omega) \subseteq L^{r}(\Omega)$ continuously (resp. compactly), if $1 \leqslant r \leqslant q^{*}\left(\right.$ resp. $\left.1 \leqslant r<q^{*}\right)$, where $q^{*}=\frac{N q}{N-q}$.

(c) $L^{p}(\Omega) \subseteq L^{\vartheta}(\Omega)$ continuously. 
Let $V: W_{0}^{1, \vartheta}(\Omega) \rightarrow W_{0}^{1, \vartheta}(\Omega)^{*}$ be the nonlinear map defined by

$$
\langle V(u), h\rangle=\int_{\Omega}\left(a(z)|D u|^{p-2}(D u, D h)_{\mathbb{R}^{N}}+|D u|^{q-2}(D u, D h)_{\mathbb{R}^{N}}\right) d z
$$

for all $u, h \in W_{0}^{1, \vartheta}(\Omega)\left(\right.$ by $\langle\cdot, \cdot\rangle$ we denote the duality brackets for the pair $\left(W_{0}^{1, \vartheta}(\Omega), W_{0}^{1, \vartheta}(\Omega)^{*}\right)$ ). This operator has the following properties (see Liu-Dai ([9], Proposition 3.1)).

Proposition 3. The operator $V: W_{0}^{1, \vartheta}(\Omega) \longrightarrow W_{0}^{1, \vartheta}(\Omega)^{*}$ is bounded (that is, maps bounded sets into bounded sets), continuous, strictly monotone (thus maximal monotone too) and of type $(S)_{+}$, that is, $V$ has the following property: "if $u_{n} \stackrel{w}{\longrightarrow} u$ in $W_{0}^{1, \vartheta}(\Omega)$ and

$$
\limsup _{n \rightarrow+\infty}\left\langle V\left(u_{n}\right), u_{n}-u\right\rangle \leqslant 0,
$$

then $u_{n} \rightarrow u$ in $W_{0}^{1, \vartheta}(\Omega) . "$

In the sequel by $\varrho_{a}$ we denote the modular function defined by

$$
\varrho_{a}(D u)=\int_{\Omega} a(z)|D u|^{p} d z \quad \forall u \in W_{0}^{1, \vartheta}(\Omega) .
$$

For every $x \in \mathbb{R}$, let

$$
x^{+}=\max \{x, 0\}, \quad x^{-}=\max \{-x, 0\} .
$$

Then for every $u \in M(\Omega)$, we set

$$
u^{ \pm}(z)=u(z)^{ \pm} \quad \forall z \in \Omega .
$$

If $u \in W_{0}^{1, \vartheta}(\Omega)$, we know that

$$
u^{ \pm} \in W_{0}^{1, \vartheta}(\Omega), \quad u=u^{+}-u^{-}, \quad|u|=u^{+}+u^{-} .
$$

By $\widehat{\lambda}_{1}(q)$ we denote the principal eigenvalue of $\left(-\Delta_{q}, W_{0}^{1, q}(\Omega)\right)$, that is, we consider the nonlinear eigenvalue problem

$$
\left\{\begin{array}{l}
-\Delta_{q} u(z)=\widehat{\lambda}|u(z)|^{q-2} u(z) \text { in } \Omega, \\
\left.u\right|_{\partial \Omega}=0 .
\end{array}\right.
$$

This problem has a smallest eigenvalue $\widehat{\lambda}_{1}(q)>0$, which has the following variational characterization

$$
\widehat{\lambda}_{1}(q)=\inf \left\{\frac{\|D u\|_{q}^{q}}{\|u\|_{q}^{q}}: u \in W_{0}^{1, q}(\Omega), u \neq 0\right\} .
$$

This eigenvalue is simple and isolated and the corresponding eigenfunctions have fixed sign and belong in $C^{1}(\bar{\Omega})$. In fact this is the only eigenvalue with eigenfunctions of fixed sign. All the other eigenvalues have eigenfunctions which are nodal. Moreover, if $C_{+}$is the positive cone of

$$
C_{0}^{1}(\bar{\Omega})=\left\{u \in C^{1}(\bar{\Omega}):\left.u\right|_{\partial \Omega}=0\right\}
$$

that is

$$
C_{+}=\left\{u \in C_{0}^{1}(\bar{\Omega}): u(z) \geqslant 0 \text { for all } z \in \bar{\Omega}\right\}
$$

and $\widehat{u} \in C_{0}^{1}(\bar{\Omega})$ is a positive eigenfunction corresponding to $\widehat{\lambda}_{1}(q)>0$, then

$$
\widehat{u} \in \operatorname{int} C_{+}=\left\{u \in C_{+}: u(z)>0 \text { for all } z \in \Omega,\left.\frac{\partial u}{\partial n}\right|_{\partial \Omega}<\right\},
$$

with $n$ being the outward unit normal on $\partial \Omega$ and $\frac{\partial u}{\partial n}=(D u, n)_{\mathbb{R}^{N}}$. 
The next lemma is an easy consequence of the above properties (see for example Mugnai-Papageorgiou ([25], Lemma 4.11)).

Lemma 1. If $\beta \in L^{\infty}(\Omega), \beta(z) \leqslant \widehat{\lambda}_{1}(q)$ for almost all $z \in \Omega$ and the inequality is strict on a set of positive Lebesgue measure, then there exists $\widetilde{c}>0$ such that

$$
\widetilde{c}\|D u\|_{q}^{q} \leqslant\|D u\|_{q}^{q}-\int_{\Omega} \beta(z)|u|^{q} d z \quad \forall u \in W_{0}^{1, q}(\Omega) .
$$

Next we introduce our hypotheses on the reaction $f(z, x)$.

Hypothesis 2. $f: \Omega \times \mathbb{R} \longrightarrow \mathbb{R}$ is a Carathéodory function such that $f(z, 0)=0$ for almost all $z \in \Omega$ and

(i) $|f(z, x)| \leqslant \widehat{a}(z)\left(1+|x|^{r-1}\right)$ for almost all $z \in \Omega$, all $x \in \mathbb{R}$ with $\widehat{a} \in L^{\infty}(\Omega), p<r<q^{*}$;

(ii) $\lim _{x \rightarrow \pm \infty} \frac{f(z, x)}{|x|^{p-2} x}=+\infty$ uniformly for almost all $z \in \Omega$;

(iii) for almost all $z \in \Omega$, the quotient function $x \longmapsto \frac{f(z, x)}{|x|^{p-1}}$ is increasing on $\mathbb{R} \backslash\{0\}$;

(iv) there exists $\widehat{\beta} \in L^{\infty}(\Omega)$ and $\widehat{c}_{0}>0$ such that $\widehat{\beta} \not \equiv \widehat{\lambda}_{1}(q)$,

$$
\widehat{\beta}(z) \leqslant \widehat{\lambda}_{1}(q) \text { for a.a. } z \in \Omega
$$

and

$$
-\widehat{c}_{0} \leq \liminf _{x \rightarrow 0} \frac{f(z, x)}{|x|^{q-2} x} \leq \limsup _{x \rightarrow 0} \frac{f(z, x)}{|x|^{q-2} x} \leq \beta(z) \quad \text { uniformly for a.a. } z \in \Omega .
$$

Remark 2. Hypothesis $H 2$ (ii) implies that for almost all $z \in \Omega, f(z, \cdot)$ is $(p-1)$-superlinear. However, we do not assume the usual in superlinear problems Ambrosetti-Rabinowitz condition (see, for example, Willem ([14], p. 46)). This condition is used by Liu-Dai [9] (see hypothesis $\left(f_{4}\right)$ in [9]). The Ambrosetti-Rabinowitz condition is a convenient but restrictive condition which permits the verification of the Palais-Smale condition (the compactness type condition on the energy functional), which is necessary in order to apply the minimax theorem of the critical point theory. Furthermore, hypothesis H2 (iii) is a relaxed version of Nehari-type monotonicity condition which requires that the quotient function $x \longmapsto \frac{f(z, x)}{|x|^{p-1}}$ is strictly increasing on $\mathbb{R} \backslash\{0\}$. This stronger version is used by Liu-Dai [9] (see hypothesis $\left(f_{5}\right)$ in [9]). Finally hypothesis H2 (iv) describes the behaviour of $f(z, \cdot)$ near zero and is less restrictive than the corresponding condition in Liu-Dai [9] (see hypothesis $\left(f_{3}\right)$ in [9]), who requires that $f(z, \cdot)$ is strictly $(q-1)$-sublinear near zero. Hypotheses $H 2$ are also less restrictive than those of Gasinski-Papageorgiou [8] (see hypotheses $H(f)$ in [8]).

Example 1. The following functions satisfy hypotheses H2, but fail to satisfy the hypotheses of Gasiński-Papageorgiou [8] and Liu-Dai [9]:

$$
\begin{aligned}
& f_{1}(x)= \begin{cases}|x|^{p-2} x & \text { if }|x| \leqslant 1, \\
|x|^{p-2} x(\ln |x|+1) & \text { if } 1<|x|,\end{cases} \\
& f_{2}(x)=\left\{\begin{array}{lll}
\beta|x|^{q-2} x+\eta|x|^{p-2} x \ln (1+|x|) & \text { if }|x| \leqslant 1, \\
|x|^{p-2} x \ln |x|+\hat{\eta}|x|^{p-2} x & \text { if } 1<|x|
\end{array}\right.
\end{aligned}
$$

with $\beta<\widehat{\lambda}_{1}(q), \eta \geqslant 2 \beta(p-q)$ and $\hat{\eta}=\beta+\eta \ln 2$. In these examples for the sake of simplicity we have dropped the $z$-dependence.

For $\eta>0$, we consider the following perturbation of the reaction

$$
f_{\eta}(z, x)=f(z, x)+\eta r|x|^{r-2} x .
$$


Note that $f_{\eta}$ satisfies the same hypotheses as $f$ and in fact now we have that for almost all $z \in \Omega$, the quotient function $x \longmapsto \frac{f_{\eta}(z, x)}{|x|^{p-1}}$ is strictly increasing on $\mathbb{R} \backslash\{0\}$.

We consider the Dirichlet double phase problem with $f_{\eta}(z, x)$ as reaction. So, we consider the following problem

$$
\left\{\begin{array}{l}
-\Delta_{p}^{a} u(z)-\Delta_{q} u(z)=f_{\eta}(z, u(z)) \quad \text { in } \Omega \\
\left.u\right|_{\partial \Omega}=0,1<q<p .
\end{array}\right.
$$

First we will prove a multiplicity result for problem $\left(P_{\eta}\right)$ and then let $\eta \rightarrow 0^{+}$to have the multiplicity theorem for the original problem $(P)$.

We set

$$
F_{\eta}(z, x)=\int_{0}^{x} f_{\eta}(z, s) d s
$$

and consider the energy (Euler) functional $\varphi_{\eta}: W_{0}^{1, \vartheta}(\Omega) \longrightarrow \mathbb{R}$ for problem $\left(P_{\eta}\right)$ defined by

$$
\varphi_{\eta}(u)=\frac{1}{p} \varrho_{a}(D u)+\frac{1}{q}\|D u\|_{q}^{q}-\int_{\Omega} F_{\eta}(z, u) d z \quad \forall u \in W_{0}^{1, \vartheta}(\Omega) .
$$

Evidently $\varphi_{\eta} \in C^{1}\left(W_{0}^{1, \vartheta}(\Omega)\right)$ and we have

$$
\left\langle\varphi_{\eta}^{\prime}(u), h\right\rangle=\langle V(u), h\rangle-\int_{\Omega} f_{\eta}(z, u) h d z \quad \forall h, u \in W_{0}^{1, \vartheta}(\Omega) .
$$

Since we want to provide sign information for all the solutions produced, we introduce also the positive and negative truncations $\varphi_{\eta}^{ \pm}: W_{0}^{1, \vartheta}(\Omega) \longrightarrow \mathbb{R}$ defined by

$$
\varphi_{\eta}^{ \pm}(u)=\frac{1}{p} \varrho_{a}(D u)+\frac{1}{q}\|D u\|_{q}^{q}-\int_{\Omega} F_{\eta}\left(z, \pm u^{ \pm}\right) d z \quad \forall u \in W_{0}^{1, \vartheta}(\Omega) .
$$

Again we have $\varphi_{\eta}^{ \pm} \in C^{1}\left(W_{0}^{1, \vartheta}(\Omega)\right)$.

We introduce the Nehari manifold $N^{\eta}$ for the functional $\varphi_{\eta}$ defined by

$$
N^{\eta}=\left\{u \in W_{0}^{1, \vartheta}(\Omega):\left\langle\varphi_{\eta}^{\prime}(u), u\right\rangle=0, u \neq 0\right\} .
$$

Evidently the Nehari manifold $N^{\eta}$ contains the nontrivial weak solutions of problem $\left(P_{\eta}\right)$. In order to produce constant sign solutions, we introduce the corresponding sets for the functionals $\varphi_{\eta}^{ \pm}$, namely the sets

$$
\begin{aligned}
& N_{+}^{\eta}=\left\{u \in W_{0}^{1, \vartheta}(\Omega):\left\langle\left(\varphi_{\eta}^{+}\right)^{\prime}(u), u\right\rangle=0, u \geqslant 0, u \neq 0\right\}, \\
& N_{-}^{\eta}=\left\{u \in W_{0}^{1, \vartheta}(\Omega):\left\langle\left(\varphi_{\eta}^{-}\right)^{\prime}(u), u\right\rangle=0, u \leqslant 0, u \neq 0\right\} .
\end{aligned}
$$

Evidently $N_{+}^{\eta}, N_{-}^{\eta} \subseteq N^{\eta}$.

Finally for the purpose of producing nodal solutions, we introduce also the set

$$
N_{0}^{\eta}=\left\{u \in W_{0}^{1, \vartheta}(\Omega): u^{+} \in N^{\eta},-u^{-} \in N^{\eta}\right\} .
$$

Given $u \in W_{0}^{1, \vartheta}(\Omega) \backslash\{0\}$, by $k_{u}^{\eta}$ we denote the corresponding fibering function defined by

$$
k_{u}^{\eta}(t)=\varphi_{\eta}(t u) \quad \forall t \geqslant 0 .
$$

Note that $t u \in N^{\eta}$ if and only if $\left(k_{u}^{\eta}\right)^{\prime}(t)=0$.

Proposition 4. If hypotheses $H 1, H 2$ hold and $u \in W_{0}^{1, \vartheta}(\Omega) \backslash\{0\}$, then there exists unique $t_{u}>0$ such that $t_{u} u \in N^{\eta}$. 
Proof. Consider the function

$$
\mu_{u}^{\eta}(t)=t^{p} \varrho_{a}(D u)+t^{q}\|D u\|_{q}^{q}-\int_{\Omega} f_{\eta}(z, t u)(t u) d z .
$$

Evidently $\mu_{u}^{\eta}(t)=t\left(k_{u}^{\eta}\right)^{\prime}(t)$ for all $t \geqslant 0$.

Note that hypothesis H2 (iii) is equivalent to saying that for almost all $z \in \Omega$ the quotient map $x \longmapsto \frac{f(z, x) x}{|x|^{p}}$ is increasing in $|x| \neq 0$. Therefore, for $x \neq 0$ and $t \in(0,1)$ we have

$$
\frac{f_{\eta}(z, t x)(t x)}{t^{p}|x|^{p}}<\frac{f_{\eta}(z, x) x}{|x|^{p}} \quad \text { for a.a. } z \in \Omega,
$$

so

$$
f_{\eta}(z, t x)(t x)<t^{p} f_{\eta}(z, x) \quad \text { for a.a. } z \in \Omega .
$$

Using (4) in (3), we have

$$
\mu_{u}^{\eta}(t) \geqslant t^{p} \varrho_{a}(D u)+t^{q}\|D u\|_{q}^{q}-t^{p} \int_{\Omega} f_{\eta}(z, u) u d z
$$

Since $q<p$, we see that there exists $t_{0} \in(0,1)$ such that

$$
\mu_{u}^{\eta}(t)>0 \text { for all } t \in\left(0, t_{0}\right)
$$

On the other hand, we have

$$
\frac{\mu_{u}^{\eta}(t)}{t^{p}}=\varrho_{a}(D u)+\frac{1}{t^{p-q}}\|D u\|_{q}^{q}-\int_{\Omega} \frac{f_{\eta}(z, t u)(t u)}{t^{p}} d z .
$$

Using hypothesis H2 (ii) we see that

$$
\lim _{t \rightarrow+\infty} \frac{\mu_{u}^{\eta}(t)}{t^{p}}=-\infty
$$

so

$$
\mu_{u}^{\eta}(t)<0 \text { for all } t>0 \text { large. }
$$

Then (5), (6) and Bolzano's theorem, imply that there exists $t_{u}>0$ such that

$$
\mu_{u}^{\eta}\left(t_{u}\right)=0,
$$

so

$$
t_{u}\left(k_{u}^{\eta}\right)^{\prime}\left(t_{u}\right)=0
$$

thus

$$
\left(k_{u}^{\eta}\right)^{\prime}\left(t_{u}\right)=0
$$

and hence $t_{u} u \in N^{\eta}$. Note that

$$
\mu_{u}^{\eta}(t)=0 \quad \Longleftrightarrow \quad \varrho_{a}(D u)=\int_{\Omega} \frac{f_{\eta}(z, t u)(t u)}{t^{p}} d z-\frac{1}{t^{p-q}}\|D u\|_{q}^{q}
$$

and observe that in the last equation the right hand side is strictly increasing in $t>0$. Therefore, $t_{u}>0$ is unique.

The Nehari manifold is much smaller than the ambient space $W_{0}^{1, \vartheta}(\Omega)$. So, $\left.\varphi_{\eta}\right|_{N^{\eta}}$ exhibits properties which fail to be true globally. In fact on account of hypothesis $\mathrm{H} 2$ (ii), $\varphi_{\eta}$ is unbounded below. However as we will show in the next proposition $\left.\varphi_{\eta}\right|_{N^{\eta}}$ is coercive, hence bounded below. This illustrates the usefulness of the Nehari manifold.

Proposition 5. If hypotheses $H 1, H 2$ hold, then $\left.\varphi_{\eta}\right|_{N^{\eta}}$ is coercive. 
Proof. We argue by contradiction. So, suppose that we can find a sequence $\left\{u_{n}\right\}_{n \in \mathbb{N}} \subseteq N^{\eta}$ such that

$$
\varphi_{\eta}\left(u_{n}\right) \leqslant c_{1} \quad \text { for all } n \in \mathbb{N} \text { and }\left\|u_{n}\right\| \rightarrow+\infty,
$$

for some $c_{1}>0$. Let $y_{n}=\frac{u_{n}}{\left\|u_{n}\right\|}$ for $n \in \mathbb{N}$. Then $\left\|y_{n}\right\|=1$ for all $n \in \mathbb{N}$ and so we may assume that

$$
y_{n} \stackrel{w}{\longrightarrow} y \quad \text { in } W_{0}^{1, \vartheta}(\Omega), \quad y_{n} \longrightarrow y \quad \text { in } L^{r}(\Omega)
$$

(see Proposition 2). First we assume that $y \neq 0$. Let

$$
\widehat{\Omega}=\{z \in \Omega:|y(z)|>0\} .
$$

Then $|\widehat{\Omega}|_{N}>0$, where by $|\cdot|_{N}$ we denote the Lebesgue measure on $\mathbb{R}^{N}$. Hence we have

$$
\left|u_{n}(z)\right| \longrightarrow+\infty \text { for a.a. } z \in \widehat{\Omega},
$$

so

$$
\frac{F_{\eta}\left(z, u_{n}(z)\right)}{\left|u_{n}(z)\right|^{p}} \longrightarrow+\infty \quad \text { for a.a. } z \in \widehat{\Omega}
$$

(see hypothesis H2 (ii)). By Fatou's lemma, we have

$$
\int_{\widehat{\Omega}} \frac{F_{\eta}\left(z, u_{n}\right)}{\left\|u_{n}\right\|^{p}} d z \longrightarrow+\infty
$$

(recall that $|\widehat{\Omega}|_{N}>0$ ). On account of hypotheses H2 (i),(ii), there exists $M>0$ such that

$$
F_{\eta}(z, x) \geqslant-M \text { for a.a. } z \in \Omega \text {, all } x \in \mathbb{R} .
$$

So, it follows that

$$
\int_{\Omega} \frac{F_{\eta}\left(z, u_{n}\right)}{\left\|u_{n}\right\|^{p}} d z \longrightarrow+\infty
$$

For $x \neq 0$ and $\tau \geqslant 0$, using the chain rule and hypothesis $\mathrm{H} 2$ (iii), we have

$$
\begin{aligned}
& \frac{1-\tau^{p}}{p} f_{\eta}(z, x) x+F_{\eta}(z, \tau x)-F_{\eta}(z, x) \\
= & \int_{\tau}^{1} f_{\eta}(z, x) x s^{p-1} d s-\int_{\tau}^{1} \frac{d}{d s} F_{\eta}(z, s x) d s \\
= & \int_{\tau}^{1} f_{\eta}(z, x) x s^{p-1} d s-\int_{\tau}^{1} f_{\eta}(z, s x) x d s \\
= & \int_{\tau}^{1}\left(\frac{f_{\eta}(z, x)}{|x|^{p-1}}-\frac{f_{\eta}(z, s x)}{(s|x|)^{p-1}}\right) s^{p-1} x|x|^{p-1} d s \\
> & 0,
\end{aligned}
$$

so

$$
F_{\eta}(z, x)-F_{\eta}(z, \tau x) \leqslant \frac{1-\tau^{p}}{p} f_{\eta}(z, x) x \text { for a.a. } z \in \Omega \text {, all } x \in \mathbb{R}, \tau \geqslant 0 .
$$

From (10) with $\tau=0$, we have

$$
p F_{\eta}(z, x) \leqslant f_{\eta}(z, x) x \text { for a.a. } z \in \Omega \text {, all } x \in \mathbb{R} .
$$

From (11) and (9) it follows that

$$
\int_{\Omega} \frac{f_{\eta}\left(z, u_{n}\right) u_{n}}{\left\|u_{n}\right\|^{p}} d z \longrightarrow+\infty
$$


Since $u_{n} \in N^{\eta}$ for all $n \in \mathbb{N}$, we have

$$
\varrho_{a}\left(D u_{n}\right)+\left\|D u_{n}\right\|_{q}^{q}=\int_{\Omega} f_{\eta}\left(z, u_{n}\right) u_{n} d z \quad \forall n \in \mathbb{N},
$$

so

$$
\int_{\Omega} \frac{f_{\eta}\left(z, u_{n}\right) u_{n}}{\left\|u_{n}\right\|^{p}} d z=\varrho_{a}\left(D y_{n}\right)+\frac{1}{\left\|u_{n}\right\|^{p-q}}\left\|D y_{n}\right\|_{q}^{q} \leqslant c_{2} \quad \forall n \in \mathbb{N},
$$

for some $c_{2}>0$. Comparing (12) and (13) we have a contradiction.

Next we assume that $y=0$. Let $\xi \geqslant 1$ and introduce

$$
v_{n}=(p \xi)^{\frac{1}{p}} y_{n} \quad \forall n \in \mathbb{N} .
$$

Evidently we have

$$
v_{n} \stackrel{w}{\longrightarrow} 0 \quad \text { in } W_{0}^{1, \vartheta}(\Omega) \quad \text { and } \quad v_{n} \longrightarrow 0 \quad \text { in } L^{r}(\Omega)
$$

(see (8) and recall that $y=0$ ). We obtain

$$
\int_{\Omega} F_{\eta}\left(z, v_{n}\right) d z \longrightarrow 0
$$

Let $t_{n} \in[0,1]$ be such that

$$
\varphi_{\eta}\left(t_{n} u_{n}\right)=\max _{0 \leqslant t \leqslant 1} \varphi_{\eta}\left(t u_{n}\right) .
$$

On account of (7), we can find $n_{0} \in \mathbb{N}$ such that

$$
0<\frac{(p \xi)^{\frac{1}{p}}}{\left\|u_{n}\right\|} \leqslant 1 \quad \forall n \geqslant n_{0} .
$$

From (15) and (16), we see that for $n \geqslant n_{0}$, we have

$$
\begin{aligned}
\varphi_{\eta}\left(t_{n} u_{n}\right) & \geqslant \varphi_{\eta}\left(v_{n}\right) \\
& \geqslant \xi \varrho_{a}\left(D y_{n}\right)+\frac{\xi^{\frac{q}{p}}}{q}\left\|D y_{n}\right\|_{q}^{q}-\int_{\Omega} F_{\eta}\left(z, v_{n}\right) d z \\
& \geqslant \frac{\xi^{\frac{q}{p}}}{q} \varrho_{\vartheta}\left(y_{n}\right)-\int_{\Omega} F_{\eta}\left(z, v_{n}\right) d z
\end{aligned}
$$

(since $p>1$ and $\xi \geqslant 1$ ), so

$$
\varphi_{\eta}\left(t_{n} u_{n}\right) \geqslant \frac{\xi^{\frac{q}{p}}}{2 q} \quad \forall n \geqslant n_{1} \geqslant n_{0}
$$

(see (14) and since $\left\|y_{n}\right\|=1$; see Proposition 1).

However, $\xi \geqslant 1$ is arbitrary. So, we infer that

$$
\varphi_{\eta}\left(t_{n} u_{n}\right) \longrightarrow+\infty \text { as } n \rightarrow+\infty .
$$

We know that

$$
\varphi_{\eta}(0)=0 \quad \text { and } \quad \varphi_{\eta}\left(u_{n}\right) \leqslant c_{1} \quad \forall n \in \mathbb{N}
$$

(see (7)). Then from (17) it follows that we can find $n_{2} \in \mathbb{N}$ such that

$$
t_{n} \in(0,1) \quad \forall n \geqslant n_{2} .
$$


From (15) and (18) we have

$$
\left.\frac{d}{d t} \varphi_{\eta}\left(t u_{n}\right)\right|_{t=t_{n}}=0
$$

so

$$
\left.\left\langle\varphi_{\eta}^{\prime}\left(t_{n} u_{n}\right), t_{n} u_{n}\right\rangle=0\right)
$$

(by the chain rule and (18)), so

$$
t_{n} u_{n} \in N^{\eta} \quad \forall n \geqslant n_{2},
$$

with $0<t_{n}<1$. However, we also have $u_{n} \in N^{\eta}$. Then on account of (19) and Proposition 4, we have a contradiction.

On account of hypotheses $\mathrm{H} 2$ (i),(ii), we can find $c_{3}>0$ such that

$$
\varphi_{\eta}(u) \leqslant \varphi_{\eta}^{ \pm}(u)+c_{3} \quad \forall u \in W^{1, \vartheta}(\Omega) .
$$

Then from Proposition 5, we infer the following corollary.

Corollary 1. If hypotheses $H 1, H 2$ hold, then $\left.\varphi_{\eta}^{ \pm}\right|_{N^{\eta}}$ are coercive.

Next we show that the elements of the Nehari manifold maximize the fibering function.

Proposition 6. If hypotheses $H 1, H 2$ hold and $u \in N^{\eta}$, then $\varphi_{\eta}(t u) \leqslant \varphi_{\eta}(u)$ for all $t \geqslant 0$.

Proof. We consider the corresponding fibering function

$$
k_{u}^{\eta}(t)=\varphi_{\eta}(t u) \quad \forall t \geqslant 0 .
$$

Recall that

$$
\left(k_{u}^{\eta}\right)^{\prime}(1)=\mu_{u}^{\eta}(1)
$$

(see (3)). Hypotheses H2 (i),(ii),(iv) imply that given $\beta>0$, we can find $c_{4}=c_{4}(\beta)>0$ such that

$$
F_{\eta}(z, x) \geqslant \frac{\beta}{p}|x|^{p}-c_{4}|x|^{q} \quad \text { for a.a. } z \in \Omega \text {, all } x \in \mathbb{R} .
$$

Therefore we have

$$
\begin{aligned}
k_{u}^{\eta}(t) & =\varphi_{\eta}(t u)=\frac{t^{p}}{p} \varrho_{a}(D u)+\frac{t^{q}}{q}\|D u\|_{q}^{q}-\int_{\Omega} F_{\eta}(z, t u) d z \\
& \leqslant \frac{t^{p}}{p}\left(\varrho_{a}(D u)-\beta\|u\|_{p}^{p}\right)+c_{5} t^{q},
\end{aligned}
$$

for some $c_{5}>0$ (see (20)). Choosing $\beta>\frac{\varrho_{a}(D u)}{\|u\|_{p}^{p}}$, we see that

$$
k_{u}^{\eta}(t) \leqslant c_{5} t^{q}-c_{6} t^{p},
$$

for some $c_{6}>0$, so

$$
k_{u}^{\eta}(t)<0 \text { for all } t>0 \text { large }
$$

(since $q<p$ ). On the other hand from hypotheses $\mathrm{H} 2$ (i),(iv), we see that given $\varepsilon>0$ we can find $c_{7}=c_{7}(\varepsilon)>0$ such that

$$
F_{\eta}(z, x) \leqslant \frac{1}{q}(\widehat{\beta}(z)+\varepsilon)|x|^{q}+c_{7}|x|^{r} \quad \text { for a.a. } z \in \Omega \text {, all } x \in \mathbb{R} \text {. }
$$


Hence we have

$$
\begin{aligned}
k_{u}^{\eta}(t) & =\varphi_{\eta}(t u) \\
& \geqslant \frac{t^{p}}{p} \varrho_{a}(D u)+\frac{t^{q}}{q}\left(\|D u\|_{q}^{q}-\int_{\Omega} \widehat{\beta}(z)|u|^{q} d z-\frac{\varepsilon}{\widehat{\lambda}_{1}(q)}\|D u\|_{q}^{q}\right)-c_{8} t^{r} \\
& \geqslant \frac{t^{p}}{p} \varrho_{a}(D u)+\frac{t^{q}}{q}\left(c_{9}-\frac{\varepsilon}{\widehat{\lambda}_{1}(q)}\right)\|D u\|_{q}^{q}-c_{8} t^{r}
\end{aligned}
$$

for some $c_{8}, c_{9}>0$ (see (22), (21) and Lemma 1).

Choosing $\varepsilon \in\left(0, \widehat{\lambda}_{1}(q) c_{9}\right)$, we have

$$
k_{u}^{\eta}(t) \geqslant c_{10} t^{p}-c_{8} t^{r},
$$

for some $c_{10}>0$. Since $p<r$, it follows that

$$
k_{u}^{\eta}>0 \quad \text { for } t \in(0,1) \text { small. }
$$

Then from (21) and (23), it follows that $k_{u}^{\eta}$ has a local maximizer $\widehat{t}_{u}>0$ and so we have

$$
\left(k_{u}^{\eta}\right)^{\prime}\left(\widehat{t}_{u}\right)=\mu_{u}^{\eta}\left(\widehat{t}_{u}\right)=0 .
$$

Since $u \in N^{\eta}$, from Proposition 4 we infer that

$$
\widehat{t}_{u}=1 \text { and this is a global maximizer of } k_{u}^{\eta}
$$

so

$$
k_{u}^{\eta}(t) \leqslant k_{u}^{\eta}(1) \quad \forall t \geqslant 0
$$

and thus

$$
\varphi_{\eta}(t u) \leqslant \varphi_{\eta}(u) \quad \forall t \geqslant 0
$$

We show that the elements of the Nehari manifold not only are nontrivial but in fact are bounded away from zero in norm.

Proposition 7. If hypotheses $\mathrm{H} 1, \mathrm{H} 2$ hold, then there exists $d_{0}>0$ such that $d_{0} \leqslant\|u\|_{r},\|u\|$ for all $u \in N^{\eta}$.

Proof. Hypotheses H2 (i),(iv) imply that given $\varepsilon>0$, we can find $c_{11}=c_{11}(\varepsilon)>0$ such that

$$
f(z, x) x \leqslant(\widehat{\beta}(z)+\varepsilon)|x|^{q}+c_{11}|x|^{r} \quad \text { for a.a. } z \in \Omega \text {, all } x \in \mathbb{R} .
$$

Given $u \in N^{\eta}$, we have

$$
\begin{aligned}
\varrho_{a}(D u)+\|D u\|_{q}^{q} & =\int_{\Omega} f(z, u) u d z \\
& \leqslant \int_{\Omega} \widehat{\beta}(z)|u|^{q} d z+\frac{\varepsilon}{\widehat{\lambda}_{1}(q)}\|D u\|_{q}^{q}+c_{11}\|u\|_{r}^{r}
\end{aligned}
$$

(see (24) and (2)), so

$$
\varrho_{a}(D u)+\left(\|D u\|_{q}^{q}-\int_{\Omega} \widehat{\beta}(z)|u|^{q}-\frac{\varepsilon}{\widehat{\lambda}_{1}(q)}\|D u\|_{q}^{q}\right) \leqslant c_{11}\|u\|_{r}^{r},
$$

thus

$$
\varrho_{a}(D u)+\left(c_{12}-\frac{\varepsilon}{\widehat{\lambda}_{1}(q)}\right)\|D u\|_{q}^{q} \leqslant c_{11}\|u\|_{r}^{r}
$$


for some $c_{12}>0$ (see Lemma 1 ).

Choosing $\varepsilon \in\left(0, \widehat{\lambda}_{1}(q) c_{12}\right)$, we obtain

$$
\varrho_{\vartheta}(u) \leqslant c_{13}\|u\|_{r}^{r}
$$

for some $c_{13}>0$, thus

$$
\min \left\{\|u\|^{q},\|u\|^{p}\right\} \leqslant c_{13}\|u\|_{r}^{r}
$$

(see Proposition 1).

Since $q<p<r$ and $W_{0}^{1, \vartheta}(\Omega) \subseteq L^{r}(\Omega)$ (see Proposition 2), we conclude that there exists $d_{0}>0$ such that

$$
d_{0} \leqslant\|u\|_{r},\|u\| \quad \forall u \in N^{\eta} .
$$

Similar results can be proved for the functionals $\varphi_{\eta}^{ \pm}$and the corresponding sets $N_{ \pm}^{\eta}$ (recall that $N_{ \pm}^{\eta} \subseteq N^{\eta}$ ).

Proposition 8. If hypotheses $\mathrm{H1}, \mathrm{H} 2 \mathrm{hold}$, then

(a) for every $u \in W_{0}^{1, \vartheta}(\Omega) \backslash\{0\}, u \geqslant 0$ (resp. $u \leqslant 0$ ) there exists unique $t_{u}^{+}>0$ (resp. $\left.t_{u}^{-}<0\right)$ such that

$$
t_{u}^{+} u \in N_{+}^{\eta}, \quad t_{u}^{-} u \in N_{-}^{\eta} ;
$$

(b) we have

$$
\begin{aligned}
& \varphi_{\eta}^{+}(t u) \leqslant \varphi_{\eta}^{+}(u) \quad \forall u \in N_{+}^{\eta}, t \geqslant 0, \\
& \varphi_{\eta}^{-}(t u) \leqslant \varphi_{\eta}^{-}(u) \quad \forall u \in N_{-}^{\eta}, t \geqslant 0 ;
\end{aligned}
$$

(c) there exist $d_{0}^{ \pm}>0$ such that

$$
\begin{array}{ll}
d_{0}^{+} \leqslant\|u\|_{r},\|u\| & \forall u \in N_{+}^{\eta}, \\
d_{0}^{-} \leqslant\|u\|_{r},\|u\| & \forall u \in N_{-}^{\eta} .
\end{array}
$$

\section{Multiple Solutions for Problem $\left(P_{\eta}\right)$}

Let

$$
m_{\eta}^{+}=\inf _{N_{+}^{\eta}} \varphi_{\eta}^{+} \quad \text { and } \quad m_{\eta}^{-}=\inf _{N_{-}^{\eta}} \varphi_{\eta}^{-} .
$$

We show that both infima are realized.

Proposition 9. If hypotheses $H 1, H 2$ hold, then there exist $u_{\eta}^{*} \in N_{+}^{\eta}$ and $\widehat{u}_{\eta} \in N_{-}^{\eta}$ such that

$$
\varphi_{\eta}^{+}\left(u_{\eta}^{*}\right)=m_{\eta}^{+} \text {and } \varphi_{\eta}^{-}\left(\widehat{u}_{\eta}\right)=m_{\eta}^{-} .
$$

Proof. We consider a minimizing sequence $\left\{u_{n}\right\}_{n \in \mathbb{N}} \subseteq N_{+}^{\eta}$ for $\left.\varphi_{\eta}^{+}\right|_{N_{+}^{\eta}}$. Therefore, we have

$$
\varphi_{\eta}^{+}\left(u_{n}\right) \searrow m_{\eta}^{+} .
$$

Corollary 1 implies that the sequence $\left\{u_{n}\right\}_{n \in \mathbb{N}} \subseteq W_{0}^{1, \vartheta}(\Omega)$ is bounded. So, by passing to a subsequence if necessary, we may assume that

$$
u_{n} \stackrel{w}{\longrightarrow} u_{\eta}^{*} \quad \text { in } W_{0}^{1, \vartheta}(\Omega) \quad \text { and } \quad u_{n} \longrightarrow u_{\eta}^{*} \quad \text { in } L^{r}(\Omega) .
$$

Since $u_{n} \in N_{+}^{\eta} \subseteq N^{\eta}$, we have

$$
\varrho_{a}\left(D u_{n}\right)+\left\|D u_{n}\right\|_{q}^{q}=\int_{\Omega} f_{\eta}\left(z, u_{n}\right) u_{n} d z, u_{n} \geqslant 0 \quad \forall n \in \mathbb{N} .
$$


From (25) and (26), we have $u_{\eta}^{*} \geqslant 0$ and we can say that $u_{\eta}^{*} \neq 0$. Indeed, if $u_{\eta}^{*}=0$, then from (26), we see that

$$
\varrho_{\vartheta}\left(u_{n}\right) \longrightarrow 0,
$$

so

$$
u_{n} \longrightarrow 0 \quad \text { in } W_{0}^{1, \vartheta}(\Omega)
$$

(see Proposition 1). However, from Proposition 8, we know that

$$
0<d_{0}^{+} \leqslant\left\|u_{n}\right\| \quad \forall n \in \mathbb{N} .
$$

Comparing (27) and (28) we have a contradiction. Therefore $u_{\eta}^{*} \neq 0, u_{\eta}^{*} \geqslant 0$.

The modular function $\varrho_{a}$ is continuous, convex, hence it is sequentially weakly lower semicontinuous and so from (25) we have

$$
\varrho_{a}\left(D u_{\eta}^{*}\right)+\left\|D u_{\eta}^{*}\right\|_{q}^{q} \leqslant \int_{\Omega} f_{\eta}\left(z, u_{\eta}^{*}\right) u_{\eta}^{*} d z .
$$

Suppose that the inequality (29) is strict, that is, we have

$$
\varrho_{a}\left(D u_{\eta}^{*}\right)+\left\|D u_{\eta}^{*}\right\|_{q}^{q}<\int_{\Omega} f_{\eta}\left(z, u_{\eta}^{*}\right) u_{\eta}^{*} d z .
$$

Consider the function

$$
\left(\mu_{u_{\mu}^{*}}^{\eta}\right)^{+}(t)=t^{p} \varrho_{a}\left(D u_{\eta}^{*}\right)+t^{q}\left\|D u_{\eta}^{*}\right\|_{q}^{q}-\int_{\Omega} f_{\eta}\left(z, t u_{\eta}^{*}\right)\left(t u_{\eta}^{*}\right) d z \quad \forall t \geqslant 0 .
$$

Furthermore, let

$$
k_{u_{\eta}^{*}}^{\eta,+}(t)=\varphi_{\eta}^{+}\left(t u_{\eta}^{*}\right) \quad \forall t \geqslant 0
$$

be the fibering function for $u_{\eta}^{*}$. Then

$$
\left(\mu_{u_{\eta}^{*}}^{\eta}\right)^{+}(t)=t\left(k_{u_{\eta}^{*}}^{\eta,+}\right)^{\prime}(t) .
$$

As in the proof of Proposition 4, exploiting hypothesis $\mathrm{H} 2$ (iv) and recalling that $q<p<r$, we see that

$$
\left(\mu_{u_{\eta}^{*}}^{\eta}\right)^{+}(t)>0 \text { for } t>0 \text { small, } \quad\left(\mu_{u_{\eta}^{*}}^{\eta}\right)^{+}(1)<0
$$

(see (30) and (31)). So, by Bolzano's theorem, we can find $\widehat{t}_{u_{\eta}^{*}} \in(0,1)$ such that

$$
\left(\mu_{u_{\eta}^{*}}^{\eta}\right)^{+}\left(\widehat{t}_{u_{\eta}^{*}}\right)=0
$$

so

$$
\widehat{t}_{u_{\eta}^{*}}\left(k_{u_{\eta}^{*}}^{\eta,+}\right)^{\prime}\left(\widehat{t}_{u_{\eta}^{*}}\right)=0
$$

thus

$$
\left(k_{u_{\eta}^{*}}^{\eta,+}\right)^{\prime}\left(\widehat{t}_{u_{\eta}^{*}}\right)=0
$$

and hence

$$
\widehat{t}_{u_{\eta}^{*}} u_{\eta}^{*} \in N_{+}^{\eta} .
$$

Therefore, we have

$$
\begin{aligned}
m_{\eta}^{*} & \leqslant \varphi_{\eta}^{+}\left(\widehat{t}_{u_{\eta}^{*}} u_{\eta}^{*}\right) \\
& =\frac{1}{p} \varrho_{a}\left(D\left(\widehat{t}_{u_{\eta}^{*}} u_{\eta}^{*}\right)\right)+\frac{1}{q}\left\|D\left(\widehat{t}_{u_{\eta}^{*}} u_{\eta}^{*}\right)\right\|_{q}^{q}-\int_{\Omega} F_{\eta}\left(z, \widehat{t}_{u_{\eta}^{*}} u_{\eta}^{*}\right) d z \\
& =\frac{1}{p}\left(\int_{\Omega} f\left(z, \widehat{t}_{u_{\eta}^{*}} u_{\eta}^{*}\right)\left(\widehat{t}_{u_{\eta}^{*}} u_{\eta}^{*}\right) d z-\left\|D\left(\widehat{t}_{u_{\eta}^{*}} u_{\eta}^{*}\right)\right\|_{q}^{q}\right)
\end{aligned}
$$




$$
\begin{aligned}
& +\frac{1}{q}\left\|D\left(\widehat{t}_{u_{\eta}^{*}} u_{\eta}^{*}\right)\right\|_{q}^{q}-\int_{\Omega} F_{\eta}\left(z, \widehat{t}_{u_{\eta}^{*}} u_{\eta}^{*}\right) d z \\
= & \frac{1}{p} \int_{\Omega}\left(f_{\eta}\left(z, \widehat{t}_{u_{\eta}^{*}} u_{\eta}^{*}\right)\left(\widehat{t}_{u_{\eta}^{*}} u_{\eta}^{*}\right)-p F_{\eta}\left(z, \widehat{t}_{u^{*}} u_{\eta}^{*}\right)\right) d z \\
& +\left(\frac{1}{q}-\frac{1}{p}\right)\left\|D\left(\widehat{t}_{u_{\eta}^{*}} u_{\eta}^{*}\right)\right\|_{q}^{q}
\end{aligned}
$$

(since $\widehat{t}_{u_{\eta}^{*}} u_{\eta}^{*} \in N_{+}^{\eta} \subseteq N^{\eta}$ ). From (10) we have

$$
\frac{s^{p}}{p} f_{\eta}(z, x) x-F_{\eta}(z, s x) \leqslant \frac{1}{p} f_{\eta}(z, x) x-F_{\eta}(z, x)
$$

for almost all $z \in \Omega$, all $x \in \mathbb{R}$, all $s \geqslant 0$. If $s \in(0,1)$, from (4), we have

$$
f_{\eta}(z, s x)(s x)<s^{p} f_{\eta}(z, x) x \text { for a.a. } z \in \Omega \text {, all } x \in \mathbb{R} \text {. }
$$

Therefore,

$$
\frac{1}{p} f_{\eta}(z, s x)(s x)-F_{\eta}(z, s x)<\frac{1}{p} f_{\eta}(z, x) x-F_{\eta}(z, x)
$$

for almost all $z \in \Omega$, all $x \in \mathbb{R}$, all $s \in(0,1)$. Using (33), (32) and (25), we obtain

$$
\begin{aligned}
m_{\eta}^{+} & <\frac{1}{p} \int_{\Omega}\left(f_{\eta}\left(z, u_{\eta}^{*}\right) u_{\eta}^{*}-p F_{\eta}\left(z, u_{\eta}^{*}\right)\right) d z+\left(\frac{1}{q}-\frac{1}{p}\right)\left\|D u_{\eta}^{*}\right\|_{q}^{q} \\
& \leqslant \liminf _{n \rightarrow+\infty}\left(\frac{1}{p} \int_{\Omega}\left(f_{\eta}\left(z, u_{n}\right) u_{n}-p F_{\eta}\left(z, u_{n}\right)\right) d z+\left(\frac{1}{q}-\frac{1}{p}\right)\left\|D u_{n}\right\|_{q}^{q}\right) \\
& =\liminf _{n \rightarrow+\infty}\left(\frac{1}{p} \varrho_{a}\left(D u_{n}\right)+\frac{1}{q}\left\|D u_{n}\right\|_{q}^{q}-\int_{\Omega} F_{\eta}\left(z, u_{n}\right) d z\right) \\
& =\liminf _{n \rightarrow+\infty} \varphi_{\eta}^{+}\left(u_{n}\right)=m_{\eta}^{+}
\end{aligned}
$$

(since $u_{n} \in N_{+}^{\eta} \subseteq N^{\eta}$ ), a contradiction.

This proves that the strict inequality (30) cannot happen and so from (29) we have

$$
\varrho_{a}\left(D u_{\eta}^{*}\right)+\left\|D u_{\eta}^{*}\right\|_{q}^{q}=\int_{\Omega} f\left(z, u_{\eta}^{*}\right) u_{\eta}^{*} d z .
$$

Since

$$
\int_{\Omega} f\left(z, u_{n}\right) u_{n} d z \longrightarrow \int_{\Omega} f\left(z, u_{\eta}^{*}\right) u_{\eta}^{*} d z
$$

(see (25)), we infer that

$$
\varrho_{\vartheta}\left(u_{n}\right) \longrightarrow \varrho_{\vartheta}\left(u_{\eta}^{*}\right) .
$$

From this and the uniform convexity of the double phase integrand $\vartheta(z, \cdot)$, we obtain

$$
\varrho_{\vartheta}\left(u_{n}-u_{\eta}^{*}\right) \longrightarrow 0
$$

(see Harjulehto-Hästö ([23], p. 65)), so

$$
u_{n} \longrightarrow u_{\eta}^{*} \quad \text { in } W_{0}^{1, \vartheta}(\Omega)
$$

(see Proposition 1). It follows that

$$
u_{\eta}^{*} \in N_{+}^{\eta} \quad \text { and } \quad m_{\eta}^{+}=\varphi_{\eta}^{+}\left(u_{\eta}^{*}\right) .
$$

Similarly, working with the set $N_{-}^{\eta}$ and the functional $\varphi_{\eta}^{-}$, we produce $\widehat{u}_{\eta} \in W_{0}^{1, \vartheta}(\Omega)$ such that

$$
\widehat{u}_{\eta} \in N_{-}^{\eta} \quad \text { and } \quad m_{\eta}^{-}=\varphi_{\eta}^{-}\left(\widehat{u}_{\eta}\right) \text {. }
$$


Reasoning as in Willem ([14], p. 74) and in Szulkin-Weth ([13], p. 611), via the quantitative deformation lemma of Willem [14], we can show that

$$
u_{\eta}^{*} \in K_{\varphi_{\eta}^{+}}=\left\{u \in W_{0}^{1, \vartheta}(\Omega):\left(\varphi_{\eta}^{+}\right)^{\prime}(u)=0\right\}
$$

(the critical set of $\varphi_{\eta}^{+}$) and

$$
\widehat{u}_{\eta} \in K_{\varphi_{\eta}^{-}}=\left\{u \in W_{0}^{1, \vartheta}(\Omega):\left(\varphi_{\eta}^{-}\right)^{\prime}(u)=0\right\}
$$

(the critical set of $\varphi_{\eta}^{-}$). So, $N_{+}^{\eta}$ is a natural constraint for $\varphi_{\eta}^{+}$, while $N_{-}^{\eta}$ is a natural constraint for $\varphi_{\eta}^{-}$(see Papageorgiou-Rădulescu-Repovš ([26], p. 425)).

Proposition 10. If hypotheses $H 1$ and $H 2$ hold and $u_{\eta}^{*} \in N_{+}^{\eta}, \widehat{u}_{\eta} \in N_{-}^{\eta}$ are as in Proposition 9, then $u_{\eta}^{*} \in K_{\varphi_{\eta}^{+}}, \widehat{u}_{\eta} \in K_{\varphi_{\eta}^{-}}$.

Proof. Let $\tau \in(0,+\infty) \backslash\{1\}$. We have

$$
\varphi_{\eta}^{+}\left(\tau u_{\eta}^{*}\right)<\varphi_{\eta}^{+}\left(u_{\eta}^{*}\right)=m_{\eta}^{+}
$$

(see Proposition 8 and recall that $u_{\eta}^{*} \in N_{+}^{\eta}$ ). Arguing by contradiction, suppose that

$$
\left(\varphi_{\eta}^{+}\right)^{\prime}\left(u_{\eta}^{*}\right) \neq 0 .
$$

Then we can find $\delta>0$ and $l>0$ such that

$$
\text { if }\left\|u-u_{\eta}^{*}\right\| \leqslant 3 \delta \text {, then }\left\|\left(\varphi_{\eta}^{+}\right)^{\prime}(u)\right\|_{*} \geqslant l \text {. }
$$

Consider the interval $D_{+}=\left(\frac{1}{2}, \frac{2}{3}\right)$ and note that

$$
m_{+}^{*}=\max _{\tau \in \partial D_{+}} \varphi_{\eta}^{+}\left(\tau u_{\eta}^{*}\right)<m_{\eta}^{+}
$$

(see (34)). We can always choose $\delta>0$ small so that

$$
\frac{l \delta}{8} \leqslant \frac{m_{\eta}^{+}-m_{+}^{*}}{4} .
$$

Then we apply the quantitative deformation lemma of Willem ([14], p. 38), with

$$
\varepsilon=\frac{l \delta}{8} \quad \text { and } \quad S=\bar{B}_{\delta}\left(u_{\eta}^{*}\right)
$$

(here $\bar{B}_{\delta}\left(u_{\eta}^{*}\right)=\left\{u \in W_{0}^{1, \vartheta}(\Omega):\left\|u-u_{\eta}^{*}\right\| \leqslant \delta\right\}$ ). In what follows for $c \in \mathbb{R}$,

$$
\left(\varphi_{\eta}^{+}\right)^{c}=\left\{u \in W_{0}^{1, \vartheta}(\Omega): \varphi_{\eta}^{+}(u) \leqslant c\right\} .
$$

So, there is a deformation $h:[0,1] \times W_{0}^{1, \vartheta}(\Omega) \longrightarrow W_{0}^{1, \vartheta}(\Omega)$ such that

- $h(1, u)=u$ if $\varphi_{\eta}^{+} \notin\left[m_{\eta}^{+}-2 \varepsilon, m_{\eta}^{+}+2 \varepsilon\right] \cap S_{3 \varepsilon}$;

- $h\left(1,\left(\varphi_{\eta}^{+}\right)^{m_{\eta}^{*}+\varepsilon} \cap \bar{B}_{\delta}\left(u_{\eta}^{*}\right)\right) \subseteq\left(\varphi_{\eta}^{+}\right)^{m_{\eta}^{+}-\varepsilon}$;

- $\varphi_{\eta}^{+}(h(1, u)) \leqslant \varphi_{\eta}^{+}(u)$ for all $u \in W_{0}^{1, \vartheta}(\Omega)$.

Then we have

$$
\max _{\tau \in \bar{D}_{+}} \varphi_{\eta}^{+}\left(h\left(1, \tau u_{\eta}^{*}\right)\right)<m_{\eta}^{+} .
$$


We show that

$$
h\left(1, D_{+} u_{\eta}^{*}\right) \cap N_{+}^{\eta} \neq \varnothing .
$$

Then this nonempty intersection and (36) lead to a contradiction. To show that this intersection is nonempty we argue as follows. Let $\zeta(\tau)=h\left(1, \tau u_{\eta}^{*}\right)$ and consider the functions

$$
\begin{aligned}
& \lambda_{0}(\tau)=\left\langle\left(\varphi_{\eta}^{+}\right)^{\prime}\left(\tau u_{\eta}^{*}\right), u_{\eta}^{*}\right\rangle, \\
& \lambda_{1}(\tau)=\frac{1}{\tau}\left\langle\left(\varphi_{\eta}^{+}\right)^{\prime}(\zeta(\tau)), \zeta(\tau)\right\rangle .
\end{aligned}
$$

Since $u_{\eta}^{*} \in N_{+}^{\eta}$, we have

$$
\begin{aligned}
& \left\langle\left(\varphi_{\eta}^{+}\right)^{\prime}\left(\tau u_{\eta}^{*}\right), u_{\eta}^{*}\right\rangle>0 \text { for } 0<\tau<1, \\
& \left\langle\left(\varphi_{\eta}^{+}\right)^{\prime}\left(\tau u_{\eta}^{*}\right), u_{\eta}^{*}\right\rangle<0 \text { for } 1<\tau
\end{aligned}
$$

(see Proposition 8).

Let $d_{B}(\cdot, \cdot, \cdot)$ denote the Brouwer degree. From Lloyd ([27], p. 20), we have

$$
d_{B}\left(\lambda_{0}, D_{+}, 0\right)=-1 .
$$

Note that from the choice of $\varepsilon>0$ and the properties of the deformation we have

$$
\left.\zeta\right|_{\partial D_{+}}=\mathrm{id} .
$$

Therefore,

$$
\left.\lambda_{0}\right|_{\partial D_{+}}=\left.\lambda_{1}\right|_{\partial D_{+}}
$$

(see (35) and the properties of the deformation $h$ ). So, by the boundary value dependence property of the degree (see Lloyd ([27], p. 25)), we have

$$
d_{B}\left(\lambda_{1}, D_{+}, 0\right)=d_{B}\left(\lambda_{0}, D_{+}, 0\right)=-1
$$

(see (37)). Then the solution property of the degree (see Lloyd ([27], p. 23)) implies that we can find $\tau \in D_{+}$such that

$$
\lambda_{1}(\tau)=0,
$$

so

$$
\zeta(\tau)=h\left(1, \tau u_{\tau}^{*}\right) \in N_{+}^{\eta},
$$

thus

$$
h\left(1, D_{+} u_{\eta}^{*}\right) \cap N_{+}^{\eta} \neq \varnothing .
$$

However, as we already stated earlier, this contradicts (36). Therefore, we must have $\left(\varphi_{\eta}^{+}\right)^{\prime}\left(u_{\eta}^{*}\right)=0$ and so $u_{\eta}^{*} \in K_{\varphi_{\eta}^{+}}$. Similarly we show that $\widehat{u}_{\eta} \in K_{\varphi_{\eta}^{-}}$. lutions.

So, for the approximate problem $\left(P_{\eta}\right)$ (with $\eta>0$ ), we can have two constant sign so-

Proposition 11. If hypotheses $H 1$ and $H 2$ hold and $\eta>0$, then problem $\left(P_{\eta}\right)$ has at least two constant sign solutions

$$
\begin{array}{ll}
u_{\eta}^{*} \in W_{0}^{1, \vartheta}(\Omega) \cap L^{\infty}(\Omega), & u_{\eta}^{*}(z)>0 \text { for a.a. } z \in \Omega, \\
\widehat{u}_{\eta} \in W_{0}^{1, \vartheta}(\Omega) \cap L^{\infty}(\Omega), & \widehat{u}_{\eta}(z)<0 \text { for a.a. } z \in \Omega .
\end{array}
$$

Proof. From Proposition 10, we already have two constant sign solutions

$$
u_{\eta}^{*} \in N_{+}^{\eta} \text { and } \widehat{u}_{\eta} \in N_{-}^{\eta} .
$$


From Theorem 3.1 of Gasiński-Winkert [15], we have

$$
u_{\eta}^{*}, \widehat{u}_{\eta} \in W_{0}^{1, \vartheta}(\Omega) \cap L^{\infty}(\Omega) .
$$

Finally Proposition 2.4 of Papageorgiou-Vetro-Vetro [19] implies that

$$
\widehat{u}_{\eta}(z)<0<u_{\eta}^{*}(z) \text { for a.a. } z \in \Omega \text {. }
$$

Using $N_{0}^{\eta}$, we can generate a nodal (sign changing) solution of problem $\left(P_{\eta}\right)$. We set

$$
m_{\eta}^{0}=\inf _{N_{0}^{\eta}} \varphi_{\eta} .
$$

Proposition 12. If hypotheses $H 1$ and $H 2$ hold and $\eta>0$, then we can find $y_{\eta}^{*} \in N_{0}^{\eta}$ such that

$$
\varphi_{\eta}\left(y_{\eta}^{*}\right)=m_{\eta}^{0}
$$

Proof. We consider a minimizing sequence $\left\{y_{n}\right\}_{n \in \mathbb{N}} \subseteq N_{0}^{\eta}$ for $\left.\varphi_{\eta}\right|_{N_{0}^{\eta}}$, that is

$$
y_{n} \in N_{0}^{\eta} \quad \forall n \in \mathbb{N} \text { and } \varphi_{\eta}\left(y_{n}\right) \searrow m_{\eta}^{0} \text {. }
$$

We have

$$
\left\{\begin{array}{l}
\varphi_{\eta}\left(y_{n}\right)=\varphi_{\eta}\left(y_{n}^{+}-y_{n}^{-}\right)=\varphi(\eta)\left(y_{n}^{+}\right)+\varphi_{\eta}\left(-y_{n}^{-}\right) \\
y_{n}^{+} \in N^{\eta}, \quad-y_{n}^{-} \in N^{\eta} \quad \forall n \in \mathbb{N} .
\end{array}\right.
$$

From Proposition 5, we have that the sequences $\left\{y_{n}^{+}\right\}_{n \in \mathbb{N}} \subseteq W_{0}^{1, \vartheta}(\Omega)$ and $\left\{y_{n}^{-}\right\}_{n \in \mathbb{N}} \subseteq$ $W_{0}^{1, \vartheta}(\Omega)$ are bounded. So, we may assume that

$$
y_{n}^{+} \stackrel{w}{\longrightarrow} y_{1}^{*} \quad \text { and } \quad y_{n}^{-} \stackrel{w}{\longrightarrow} y_{2}^{*} \quad \text { in } W_{0}^{1, \rho}(\Omega)
$$

Suppose that $y_{1}^{*}=0$. Since $y_{n}^{+} \in N^{\eta}$, for $n \in \mathbb{N}$ (see (39)), we have

$$
\varrho_{a}\left(D y_{n}^{+}\right)+\left\|D y_{n}^{+}\right\|_{q}^{q}=\int_{\Omega} f_{\eta}\left(z, y_{n}^{+}\right) y_{n}^{+} d z \quad \forall n \in \mathbb{N},
$$

so

$$
\varrho_{\theta}\left(y_{n}^{+}\right) \longrightarrow 0
$$

(see (40) and recall that $y_{1}^{*}=0$ ), thus

$$
y_{n}^{+} \longrightarrow 0 \quad \text { in } W_{0}^{1, \vartheta}(\Omega)
$$

(see Proposition 1).

This then contradicts Proposition 7. Therefore $y_{1}^{*} \neq 0$.

Similarly we show that $y_{2}^{*} \neq 0$.

From Proposition 4, we know that there exist unique $t_{+}, t_{-}>0$ such that

$$
t_{+} y_{1}^{*} \in N^{\eta}, \quad t_{-} y_{2}^{*} \in N^{\eta} .
$$

We set

$$
y_{\eta}^{*}=t_{+} y_{1}^{*}-t_{-} y_{2}^{*} \in W_{0}^{1, \vartheta}(\Omega),
$$

so

$$
\left(y_{\eta}^{*}\right)^{+}=t_{+} y_{1}^{*} \text { and }\left(y_{\eta}^{*}\right)^{-}=t_{-} y_{2}^{*} .
$$


Then, using (38), (39) and Proposition 6, we have

$$
\begin{aligned}
m_{\eta}^{0} & =\lim _{n \rightarrow+\infty} \varphi_{\eta}\left(y_{n}\right) \\
& =\lim _{n \rightarrow+\infty}\left(\varphi_{\eta}\left(y_{n}^{+}\right)+\varphi_{\eta}\left(-y_{n}^{-}\right)\right) \\
& \geqslant \liminf _{n \rightarrow+\infty}\left(\varphi_{\eta}\left(t_{n} y_{n}^{+}\right)+\varphi_{\eta}\left(-t_{-} y_{n}^{-}\right)\right) \\
& \geqslant \varphi_{\eta}\left(t_{n} y_{1}^{*}\right)+\varphi_{\eta}\left(-t_{-} y_{2}^{*}\right) \\
& =\varphi_{\eta}\left(y_{\eta}^{*}\right) \\
& \geqslant m_{\eta}^{0}
\end{aligned}
$$

(since $y_{\eta}^{*} \in N_{0}^{\eta}$ ), so

$$
m_{\eta}^{0}=\varphi_{\eta}\left(y_{\eta}^{*}\right)
$$

with $y_{\eta}^{*} \in N_{0}^{\eta}$.

Arguing as in the proof of Proposition 10, we show that $N_{0}^{\eta}$ is a natural constraint for the functional $\varphi_{\eta}$, that is, $y_{\eta}^{*} \in K_{\varphi_{\eta}}$. In this case we replace the interval $D_{+}$by the square

$$
\widehat{D}=\left(\frac{1}{2}, \frac{3}{2}\right) \times\left(\frac{1}{2}, \frac{3}{2}\right)
$$

and the functionals $\zeta, \lambda_{0}, \lambda_{1}$ by

$$
\begin{aligned}
\widehat{\zeta}(\tau, t) & =h\left(1, \tau y_{1}^{*}-t y_{2}^{*}\right) \quad \forall \tau, t \geqslant 0, \\
\widehat{\lambda}_{0}(\tau, t) & =\left(\left\langle\varphi_{\eta}^{\prime}\left(\tau y_{1}^{*}\right), y_{1}^{*}\right\rangle,\left\langle\varphi_{\eta}^{\prime}\left(-t y_{2}^{*}\right),-y_{2}^{*}\right\rangle\right), \\
\widehat{\lambda}_{1}(\tau, t) & =\left(\frac{1}{\tau}\left\langle\varphi_{\eta}^{\prime}\left(\zeta(\tau, t)^{+}\right), \zeta(\tau, t)^{+}\right\rangle, \frac{1}{\tau}\left\langle\varphi_{\eta}^{\prime}\left(-\zeta(\tau, t)^{-}\right),-\zeta(\tau, t)^{-}\right\rangle\right) .
\end{aligned}
$$

Reasoning as in the proof of Proposition 10, via the quantitative deformation lemma of Willem ([14], p. 38), we obtain the following result showing that $N_{0}^{\eta}$ is a natural constraint for the functional $\varphi_{\eta}$.

Proposition 13. If hypotheses $\mathrm{H} 1$ and $\mathrm{H} 2$ hold and $\eta>0$, then

$$
y_{\eta}^{*} \in K_{\varphi_{\eta}}=\left\{y \in W_{0}^{1, \vartheta}(\Omega): \varphi_{\eta}^{\prime}(y)=0\right\}
$$

and so $y_{\eta}^{*} \in W_{0}^{1, \vartheta}(\Omega) \cap L^{\infty}(\Omega)$ is a nodal solution of $\left(P_{\eta}\right)$.

Summarizing the situation for problem $\left(P_{\eta}\right)$, we can state the following multiplicity result.

Proposition 14. If hypotheses $\mathrm{H} 1$ and $\mathrm{H} 2$ hold and $\eta>0$, then problem $\left(P_{\eta}\right)$ has at least three nontrivial solutions

$$
\begin{aligned}
& u_{\eta}^{*} \in W_{0}^{1, \vartheta}(\Omega) \cap L^{\infty}(\Omega), \quad u_{\eta}^{*}(z)>0 \text { for a.a. } z \in \Omega, \\
& \widehat{u}_{\eta} \in W_{0}^{1, \vartheta}(\Omega) \cap L^{\infty}(\Omega), \quad \widehat{u}_{\eta}(z)<0 \text { for a.a. } z \in \Omega, \\
& y_{\eta}^{*} \in W_{0}^{1, \vartheta}(\Omega) \cap L^{\infty}(\Omega) \text { nodal }
\end{aligned}
$$

and

$$
\varphi_{\eta}^{+}\left(u_{\eta}^{*}\right)=m_{+}^{\eta}<0<m_{-}^{\eta}=\varphi_{\eta}^{-}\left(\widehat{u}_{\eta}\right), \quad \varphi_{\eta}\left(y_{\eta}^{*}\right)=m_{0}^{\eta} .
$$

\section{Multiple Solutions for Problem $(P)$}

In this section, we use the solutions from Proposition 14 and let $\eta \rightarrow 0^{+}$to produce multiple solutions with sign information for problem $(P)$. 
Proposition 15. If hypotheses $H 1$ and $H 2$ hold, then there exists $d^{*}>0$ such that

$$
0<d^{*} \leqslant \varphi_{\eta}(u) \quad \forall u \in N^{\eta}, \eta \in(0,1) .
$$

Proof. On account of hypotheses H2 (i),(iv), given $\varepsilon>0$, we can find $c_{14}=c_{14}(\varepsilon)>0$ such that

$$
F(z, x) \leqslant \frac{1}{q}(\widehat{\beta}(z)+\varepsilon)|x|^{q}+\frac{c_{14}}{r}|x|^{r} \quad \text { for a.a. } z \in \Omega, \text { all } x \in \mathbb{R} .
$$

If $u \in N^{\eta}(\eta \in(0,1))$, then from Proposition 6, we have

$$
\begin{aligned}
\varphi_{\eta}(u) & =\max _{t \geqslant 0} \varphi_{\eta}(t u) \\
& \geqslant \max _{t \geqslant 0}\left(\frac{t^{p}}{p} \varrho_{a}(D u)+\frac{t^{q}}{q}\|D u\|_{q}^{q}-\int_{\Omega} F(z, t u) d z-\frac{t^{r}}{r}\|u\|_{r}^{r}\right) \\
& \geqslant \max _{t \geqslant 0}\left(c_{15} t^{p}-c_{16} t^{r}\right),
\end{aligned}
$$

for some $c_{15}, c_{16}>0$ (recall that $\eta \in(0,1)$, use (41), Lemma 1 and choose $\varepsilon>0$ small), so

$$
\varphi_{\eta}(u) \geqslant d^{*}>0 \quad \forall \eta \in(0,1)
$$

(since $p<r$ ).

In a similar fashion, using Proposition 8, we obtain the following result.

Proposition 16. If hypotheses $\mathrm{H} 1$ and $\mathrm{H} 2$ hold, then there exists $d_{ \pm}^{*}>0$ such that

$$
\begin{array}{ll}
0<d_{+}^{*} \leqslant \varphi_{\eta}^{+}(u) & \forall u \in N_{+}^{\eta}, \eta \in(0,1), \\
0<d_{-}^{*} \leqslant \varphi_{\eta}^{-}(u) & \forall u \in N_{-}^{\eta}, \eta \in(0,1) .
\end{array}
$$

Now we are ready to pass to the limit as $\eta \rightarrow 0^{+}$and have the multiplicity result for problem $(P)$.

In the sequel $\varphi: W_{0}^{1, \vartheta}(\Omega) \longrightarrow \mathbb{R}$ is the energy functional for problem $(P)$ defined by

$$
\varphi(u)=\frac{1}{p} \varrho_{a}(D u)+\frac{1}{q}\|D u\|_{q}^{q}-\int_{\Omega} F(z, u) d z \quad \forall u \in W_{0}^{1, \vartheta}(\Omega) .
$$

We have that $\varphi \in C^{1}\left(W_{0}^{1, \vartheta}(\Omega)\right)$.

Furthermore, we consider the positive and negative truncations of $\varphi$, namely the $C^{1}$-functionals $\varphi_{\varphi}: W_{0}^{1, \vartheta}(\Omega) \longrightarrow \mathbb{R}$ defined by

$$
\varphi_{ \pm}(u)=\frac{1}{p} \varrho_{q}(D u)+\frac{1}{q}\|D u\|_{q}^{q}-\int_{\Omega} F\left(z, \pm u^{ \pm}\right) d z \quad \forall u \in W_{0}^{1, \vartheta}(\Omega) .
$$

Again we have $\varphi_{ \pm} \in C^{1}\left(W_{0}^{1, \vartheta}(\Omega)\right)$.

We introduce the corresponding Nehari manifolds

$$
\begin{aligned}
N & =\left\{u \in W_{0}^{1, \vartheta}(\Omega):\left\langle\varphi^{\prime}(u), u\right\rangle=0, u \neq 0\right\}, \\
N_{+} & =\left\{u \in W_{0}^{1, \vartheta}(\Omega):\left\langle\varphi_{+}^{\prime}(u), u\right\rangle=0, u \geqslant 0, u \neq 0\right\}, \\
N_{-} & =\left\{u \in W_{0}^{1, \vartheta}(\Omega):\left\langle\varphi_{-}^{\prime}(u), u\right\rangle=0, u \leqslant 0, u \neq 0\right\}, \\
N_{0} & =\left\{u \in W_{0}^{1, \vartheta}(\Omega): u^{+} \in N,-u^{-} \in N\right\} .
\end{aligned}
$$


Theorem 1. If hypotheses $H 1$ and $H 2$ hold, then problem $(P)$ has at least three nontrivial solutions

$$
\begin{aligned}
& u^{*} \in W_{0}^{1, \vartheta}(\Omega) \cap L^{\infty}(\Omega), u^{*}(z)>0 \text { for a.a. } z \in \Omega, \\
& \widehat{u} \in W_{0}^{1, \vartheta}(\Omega) \cap L^{\infty}(\Omega), \widehat{u}(z)<0 \text { for a.a. } z \in \Omega, \\
& y^{*} \in W_{0}^{1, \vartheta}(\Omega) \cap L^{\infty}(\Omega) \text { nodal. }
\end{aligned}
$$

Proof. Let $\eta_{n} \rightarrow 0^{+}$. According to Proposition 14, we can find $\bar{u}_{n}=\bar{u}_{\eta_{n}} \in N_{0}^{\eta_{n}}$ such that

$$
\varphi_{\eta_{n}}\left(\bar{u}_{n}\right)=m_{0}^{\eta_{n}} \longrightarrow m_{*}>0 \quad \text { and } \quad \varphi_{\eta_{n}}^{\prime}\left(\bar{u}_{n}\right)=0 \quad \forall n \in \mathbb{N}
$$

(see Proposition 15).

Claim. The sequence $\left\{\bar{u}_{n}\right\}_{n \in \mathbb{N}} \subseteq W_{0}^{1, \vartheta}(\Omega)$ is bounded.

We argue by contradiction. So, suppose that at least for a subsequence, we have

$$
\left\|\bar{u}_{n}\right\| \longrightarrow+\infty \text {. }
$$

Let $\bar{v}_{n}=\frac{\bar{u}_{n}}{\left\|\bar{u}_{n}\right\|}$ for $n \in \mathbb{N}$. Then $\left\|\bar{v}_{n}\right\|=1$ for all $n \in \mathbb{N}$ and so we may assume that

$$
\bar{v}_{n} \stackrel{w}{\longrightarrow} \bar{v} \quad \text { in } W_{0}^{1, \vartheta}(\Omega) \text { and } \quad \bar{v}_{n} \longrightarrow \bar{v} \quad \text { in } L^{r}(\Omega)
$$

First suppose that $\bar{v}=0$. Using (42) and Proposition 6 , for $k>1$, we have

$$
\begin{aligned}
m_{0}^{\eta_{n}} & =\varphi_{\eta_{n}}\left(\bar{u}_{n}\right) \\
& \geqslant \varphi_{\eta_{n}}\left(\frac{k}{\left\|\bar{u}_{n}\right\|} \bar{u}_{n}\right) \\
& =\varphi_{\eta_{n}}\left(k \bar{v}_{n}\right) \\
& =\frac{k^{p}}{p} \varrho_{a}\left(D \bar{v}_{n}\right)+\frac{k^{q}}{q}\left\|D \bar{v}_{n}\right\|_{q}^{q}-\int_{\Omega} F\left(z, k \bar{v}_{n}\right) d z-\eta k^{r}\left\|\bar{v}_{n}\right\|_{r}^{r} \\
& \geqslant \frac{k^{q}}{p}-\int_{\Omega} F\left(z, k \bar{v}_{n}\right) d z-\eta k^{r}\left\|\bar{v}_{n}\right\|_{r}^{r}
\end{aligned}
$$

(since $k>0, q<p,\left\|\bar{v}_{n}\right\|=1$; see Proposition 1 ).

We pass to the limit as $n \rightarrow+\infty$ and use (44) (recalling that $\bar{v}=0$ ). Then

$$
m_{*} \geqslant \frac{k^{q}}{p}>0
$$

(see (42)).

Since $k>1$ is arbitrary, we let $k \rightarrow+\infty$ and have a contradiction.

Next suppose that $\bar{v} \neq 0$ and set

$$
\widehat{\Omega}=\{z \in \Omega: v(z) \neq 0\} .
$$

We have $|\widehat{\Omega}|_{N}>0$ (here $|\cdot|_{N}$ denotes the Lebesgue measure on $\mathbb{R}^{N}$ ) and $\left|u_{n}(z)\right| \rightarrow+\infty$ for a.a. $z \in \widehat{\Omega}$. From (42) we have

$$
\begin{aligned}
0 & <\frac{m_{0}^{\eta_{n}}}{\left\|\bar{u}_{n}\right\|^{p}}=\frac{\varphi_{\eta_{n}}\left(\bar{u}_{n}\right)}{\left\|\bar{u}_{n}\right\|^{p}} \\
& \leqslant \frac{1}{p} \varrho_{a}\left(D \bar{v}_{n}\right)+\frac{1}{q\left\|\bar{u}_{n}\right\|^{p-q}}\left\|D \bar{v}_{n}\right\|_{q}^{q}-\int_{\Omega} \frac{F\left(z, \bar{u}_{n}\right)}{\left\|\bar{u}_{n}\right\|^{p}} d z \\
& \leqslant \frac{1}{q}\left(\varrho_{a}\left(D \bar{v}_{n}\right)+\left\|D \bar{v}_{n}\right\|_{q}^{q}\right)-\int_{\Omega} \frac{F\left(z, \bar{u}_{n}\right)}{\left\|\bar{u}_{n}\right\|^{p}} d z \\
& =\frac{1}{q} \varrho_{\vartheta}\left(\bar{v}_{n}\right)-\int_{\Omega} \frac{F\left(z, \bar{u}_{n}\right)}{\left\|\bar{u}_{n}\right\|^{p}} d z
\end{aligned}
$$




$$
=\frac{1}{q}-\int_{\Omega} \frac{F\left(z, \bar{u}_{n}\right)}{\left\|\bar{u}_{n}\right\|^{p}} d z
$$

for all $n \geqslant n_{1}$ (see (43) and recall that $q<p$ ).

Hypothesis H2 (ii) implies that

$$
\int_{\widehat{\Omega}} \frac{F\left(z, \bar{u}_{n}\right)}{\left\|\bar{u}_{n}\right\|^{p}} d z \longrightarrow+\infty
$$

and so

$$
\int_{\Omega} \frac{F\left(z, \bar{u}_{n}\right)}{\left\|\bar{u}_{n}\right\|^{p}} d z \longrightarrow+\infty .
$$

Passing to the limit as $n \rightarrow+\infty$ in (45), we have a contradiction. This proves the Claim.

On account of the Claim, we may assume that

$$
\bar{u}_{n} \stackrel{w}{\longrightarrow} y^{*} \quad \text { in } W_{0}^{1, \vartheta}(\Omega) \text { and } \quad \bar{u}_{n} \longrightarrow y^{*} \quad \text { in } L^{r}(\Omega) \text {. }
$$

From (42), we have

$$
\left\langle V\left(\bar{u}_{n}\right), h\right\rangle=\int_{\Omega} f_{\eta}\left(z, \bar{u}_{n}\right) h d z \quad \forall h \in W_{0}^{1, \vartheta}(\Omega)
$$

We choose $h=\bar{u}_{n}-y^{*} \in W_{0}^{1, \vartheta}(\Omega)$, pass to the limit as $n \rightarrow+\infty$ and use (46). We obtain

$$
\lim _{n \rightarrow+\infty}\left\langle V\left(\bar{u}_{n}\right), \bar{u}_{n}-y^{*}\right\rangle=0,
$$

so

$$
\bar{u}_{n} \longrightarrow y^{*} \quad \text { in } W_{0}^{1, \vartheta}(\Omega)
$$

(see Proposition 3), so

$$
\bar{u}_{n}^{+} \longrightarrow\left(y^{*}\right)^{+} \text {and } \quad \bar{u}_{n}^{-} \longrightarrow\left(y^{*}\right)^{-} \quad \text { in } W_{0}^{1, \vartheta}(\Omega) .
$$

From Proposition 15, we have

$$
\varphi(0)=0<d^{*} \leqslant \varphi\left(\left(y^{*}\right)^{ \pm}\right)
$$

(see (47) and recall that $\eta_{n} \rightarrow 0^{+}$), so $\left(y^{*}\right)^{ \pm} \neq 0$ and thus $y^{*} \in N_{0}$ (see (47)).

Moreover, we have

$$
m_{*}=\varphi\left(y_{*}\right) \geqslant m_{0}=\inf _{N_{0}} \varphi .
$$

We will show that in fact equality holds in (48). To this end, given $\varepsilon>0$ we choose $v_{\varepsilon} \in N_{0}$ such that

$$
\varphi\left(v_{\varepsilon}\right) \leqslant m_{0}+\varepsilon .
$$

According to Proposition 8 , we can find $\tau_{n}, t_{n}>0$ such that

$$
\tau_{n} v_{\mathcal{\varepsilon}}^{+} \in N_{+}^{\eta_{n}},-t_{n} v_{\mathcal{\varepsilon}}^{-} \in N_{-}^{\eta_{n}}, \quad \text { hence } \tau_{n} v_{\varepsilon}^{+}-t_{n} v_{\mathcal{\varepsilon}}^{-} \in N_{-}^{\eta_{n}} \quad \forall n \in \mathbb{N} .
$$

We know that

$$
\varphi(u)=\varphi_{\eta}(u)+\eta\|u\|_{r}^{r} \quad \forall u \in W_{0}^{1, \vartheta}(\Omega), \eta>0
$$

Then,

$$
\begin{aligned}
m_{0}+\varepsilon & \geqslant \varphi\left(v_{\varepsilon}\right)=\varphi\left(v_{\mathcal{\varepsilon}}^{+}\right)+\varphi\left(-v_{\mathcal{\varepsilon}}^{-}\right) \\
& \geqslant \varphi\left(\tau_{n} v_{\mathcal{\varepsilon}}^{+}\right)+\varphi\left(-t_{n} v_{\mathcal{\varepsilon}}^{-}\right) \\
& =\varphi\left(\tau_{n} v_{\mathcal{\varepsilon}}^{+}-t_{n} v_{\mathcal{\varepsilon}}^{-}\right) \\
& \geqslant \varphi_{\eta_{n}}\left(\tau_{n} v_{\mathcal{\varepsilon}}^{+}-t_{n} v_{\mathcal{\varepsilon}}^{-}\right)
\end{aligned}
$$




$$
\geqslant m_{0}^{\eta_{n}} \quad \forall n \in \mathbb{N}
$$

(see Proposition 6, (51), (50)), so

$$
m_{0}+\varepsilon \geqslant m_{*}
$$

(see (42)). Since $\varepsilon>0$ is arbitrary, we let $\varepsilon \rightarrow 0^{+}$and obtain

$$
m_{*} \leqslant m_{0}
$$

so

$$
m_{*}=m_{0}
$$

(see (48)). From (48), we have

$$
\varphi\left(y^{*}\right)=m_{0}, \quad y^{*} \in N_{0} .
$$

Since $\varphi_{\eta_{n}}^{\prime}\left(\bar{u}_{n}\right)=0$ for all $n \in \mathbb{N}$, we have $\varphi^{\prime}\left(y^{*}\right)=0$ and so we conclude that $y^{*}$ is nodal solution of $(P)$ and $y^{*} \in W_{0}^{1, \vartheta}(\Omega) \cap L^{\infty}(\Omega)$.

Similarly, using Propositions 14 and 16, we produce $u^{*} \in N_{+}$and $\widehat{u} \in N_{-}$with

$$
\begin{aligned}
& \varphi_{+}\left(u^{*}\right)=m_{+}=\inf _{N_{+}} \varphi, u^{*} \in W_{0}^{1, \vartheta}(\Omega) \cap L^{\infty}(\Omega), u^{*}(z)>0 \text { a.e. in } \Omega, \\
& \varphi_{-}(\widehat{u})=m_{-}=\inf _{N_{-}} \varphi, \widehat{u} \in W_{0}^{1, \vartheta}(\Omega) \cap L^{\infty}(\Omega), \widehat{u}(z)<0 \text { a.e. in } \Omega .
\end{aligned}
$$

\section{Conclusions}

We have extended the classical multiplicity result to superlinear problems (see [10,25], three solutions theorem) to double-phase problems, under more general conditions on the reaction and we provided sign information for all the solutions produced.

Author Contributions: Conceptualization, methodology, validation, and formal analysis: B.D., L.G. and N.S.P.; writing-original draft preparation: N.S.P., review and editing: N.S.P. and L.G.; visualization: L.G.; supervision: N.S.P.; project administration: N.S.P. All authors have read and agreed to the published version of the manuscript.

Funding: This research received no external funding.

Institutional Review Board Statement: Not applicable.

Informed Consent Statement: Not applicable.

Data Availability Statement: Not applicable.

Acknowledgments: The authors wish to thank the three anonymous referees for their remarks.

Conflicts of Interest: The authors declare no conflict of interest.

\section{References}

1. Mingione, G.; Rădulescu, V. Recent developments in problems with nonstandard growth and nonuniform ellipticity. J. Math. Anal. Appl. 2021, 501, 125197. [CrossRef]

2. Marcellini, P. Regularity of minimizers of integrals of the calculus of variations with nonstandard growth conditions. Arch. Rational Mech. Anal. 1989, 105, 267-284. [CrossRef]

3. Marcellini, P. Regularity and existence of solutions of elliptic equations with $p, q$-growth conditions. J. Differ. Equ. 1991, 90, 1-30. [CrossRef]

4. Zhikov, V.V. Averaging of functionals of the calculus of variations and elasticity theory. Izv. Akad. Nauk SSSR Ser. Mat. 1986, 50, 675-710. [CrossRef]

5. Zhikov, V.V. On variational problems and nonlinear elliptic equations with nonstandard growth conditions, Problems in mathematical analysis. No. 54. J. Math. Sci. 2011, 173, 463-570. [CrossRef]

6. Baroni, P.; Colombo, M.; Mingione, G. Regularity for general functionals with double phase. Calc. Var. Partial Differ. Equ. 2018, 57, 48. [CrossRef] 
7. Ragusa, M.A.; Tachikawa, A. Regularity for minimizers for functionals of double phase with variable exponents. Adv. Nonlinear Anal. 2020, 9, 710-728. [CrossRef]

8. Gasiński, L.; Papageorgiou, N.S. Constant sign and nodal solutions for superlinear double phase problems. Adv. Calc. Var. 2019. [CrossRef]

9. Liu, W.; Dai, G. Existence and multiplicity results for double phase problem. J. Differ. Equ. 2018, 265, 4311-4334. [CrossRef]

10. Wang, Z.Q. On a superlinear elliptic equation. Ann. Inst. H. Poincaré Anal. Non Linéaire 1991, 8, 43-57. [CrossRef]

11. Papageorgiou, N.S.; Rădulescu, V.D. Multiplicity theorems for resonant and superlinear nonhomogeneous elliptic equations. Topol. Methods Nonlinear Anal. 2016, 48, 283-320. [CrossRef]

12. Brown, K.J.; Wu, T.-F. A fibering map approach to a semilinear elliptic boundary value problem. Electron. Differ. Equ. 2007, 69, 9.

13. Szulkin, A.; Weth, T. The Method of Nehari Manifold. In Handbook of Nonconvex Analysis and Applications; Int. Press: Somerville, MA, USA, 2010; pp. 597-632.

14. Willem, M. Minimax Theorems. In Progress in Nonlinear Differential Equations and their Applications, 24; Birkhäuser Boston, Inc.: Boston, MA, USA, 1996.

15. Gasiński, L.; Winkert, P. Constant sign solutions for double phase problems with superlinear nonlinearity. Nonlinear Anal. 2020, 195, 111739. [CrossRef]

16. Colasuonno, F.; Squassina, M. Eigenvalues for double phase variational integrals. Ann. Mat. Pura Appl. 2016, 195, 1917-1959. [CrossRef]

17. Ge, B.; Wang, L.-Y.; Lu, J.-F. On a class of double-phase problem without Ambrosetti-Rabinowitz-type conditions. Appl. Anal. 2019, 100, 2147-2162. [CrossRef]

18. Gasiński, L.; Winkert, P. Sign changing solution for a double phase problem with nonlinear boundary condition via the Nehari manifold. J. Differ. Equ. 2021, 274, 1037-1066. [CrossRef]

19. Papageorgiou, N.S.; Vetro, C.; Vetro, F. Multiple solutions for parametric double phase Dirichlet problems. Commun. Contemp. Math. 2021, 23, 2050006. [CrossRef]

20. Kalyabin, D.V.; Sadovnikov, A.V.; Beginin, E.N.; Nikitov, S.A. Surface spin waves propagation in tapered magnetic stripe. J. Appl. Phys. 2019, 126, 173907. [CrossRef]

21. Sadovnikov, A.V.; Odintsov, S.A.; Beginin, E.N.; Grachev, A.A.; Gubanov, V.A.; Sheshukova, S.E.; Sharaevskii, Y.P.; Nikitov, S.A. Nonlinear spin wave effects in the system of lateral magnonic structures. JETP Lett. 2018, 107, 25-29. [CrossRef]

22. Rădulescu, V.D. Isotropic and anistropic double-phase problems: Old and new. Opusc. Math. 2019, 39, 259-279. [CrossRef]

23. Harjulehto, P.; Hästö, P. Orlicz Spaces and Generalized Orlicz Spaces. In Lecture Notes in Mathematics 2236; Springer: Cham, Switzerland, 2019.

24. Adams, R.A. Sobolev Spaces; Academic Press: New York, NY, USA; London, UK, 1975.

25. Mugnai, D.; Papageorgiou, N.S. Resonant nonlinear Neumann problems with indefinite weight. Ann. Sc. Norm. Super. Pisa Cl. Sci. 2012, 11, 729-788.

26. Papageorgiou, N.S.; Rădulescu, V.D.; Repovš, D.D. Nonlinear Analysis-Theory and Methods. In Springer Monographs in Mathematics; Springer: Cham, Switzerland, 2019.

27. Lloyd, N.G. Degree Theory, Cambridge Tracts in Mathematics, No. 73; Cambridge University Press: Cambridge, UK; New York, NY, USA; Melbourne, Australia, 1978. 